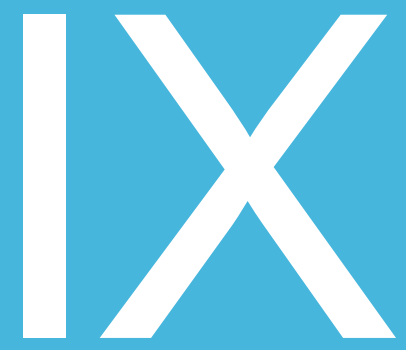

\title{
Las relaciones públicas y la
} responsabilidad social de las

\section{universidades peruanas según la nueva ley universitaria}

\section{Public relations and social responsibility of the Peruvian universities according to the new university law \\ Universidad Peruana Unión}

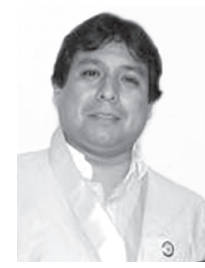

\section{István János Kovács Halay}

Doctor en Gestión Educativa. Magíster en Administración Educativa por la Universidad Peruana Unión. Licenciado en Ciencias de la Comunicación por la Universidad de San Martín de Porres. Es director regional por el Perú de la Red Iberoamericana de Relaciones Públicas (REDIRP) con sede en Buenos Aires - Argentina. Actualmente se desempeña como docente de posgrado en la Maestría en Educación, Doctorado en Administración y en la Escuela Académico Profesional de Ciencias de la Comunicación en la Universidad Peruana Unión (UPeU). Es columnista internacional en temas de Relaciones Públicas de la Revista Internacional Negocios y Management de Argentina y del portal RP- Bahía del Brasil. 


\section{Resumen}

La nueva ley universitaria 30220 promulgada el 8 de julio de 2014 obliga a las universidades a que replanteen su Responsabilidad Social obligándolas a desarrollar auténticos programas de proyección social que permitan que diversos sectores de la sociedad puedan tener el conocimiento tecnológico para poder auto gestionar su desarrollo mediante la producción de nuevos conocimientos que estén acordes con nuestra realidad nacional multicultural. Las universidades deben desarrollar labores de Relaciones Públicas estratégicas consistentes en fomentar canales de comunicación con la sociedad en donde ambas partes sintonicen intereses y participen en conjunto de manera democrática en la concepción, desarrollo y evaluación de dichos programas de Responsabilidad Social fomentando de esta manera la integración armoniosa de ambas partes. Además de ello es importante que las universidades informen con transparencia la manera de gestionar sus recursos y de esta manera generar confianza en la sociedad. Para cubrir con las múltiples necesidades de nuestro país, las universidades deben celebrar convenios con otras universidades y empresas en general para ofrecer mejores oportunidades de desarrollo profesional y humano a toda la comunidad que las conforman. La Responsabilidad Social universitaria dejó de ser una simple ayuda caritativa para los sectores más deprimidos de la sociedad. La nueva ley obliga a que las universidades hagan historia en la sociedad formando una mentalidad empresarial entre sus docentes, estudiantes y egresados para de esta manera lograr el progreso nacional con equidad, hecho que contribuirá al desarrollo conjunto de las universidades con la sociedad.

Palabras clave: Nueva ley universitaria, universidad, Responsabilidad Social, Relaciones Públicas.

\section{Abstract}

The new university law 30220, promulgated on July 8,2014 , obliges universities to rethink their social responsibility in order to develop genuine programs of social projection that allow various sectors of society to have the technical knowledge to self-manage their development through the production of new knowledge that are consistent with our multicultural national reality. Universities should develop work Strategic Public Relations consistent in promoting communication channels with the society in which both parties tune interests and participate together in a democratic manner in the design, development and evaluation of such programs Social Responsibility thus promoting integration harmonious on both sides. In addition, it is important that universities report transparently how to manage their resources and thus build confidence in society. To meet the multiple needs of our country, universities must enter into agreements with other universities and companies generally offer better opportunities for professional and personal the whole community that shape development. Social Responsibility university ceased to be a simple charitable assistance to the poorest sectors of society. The new law requires universities to make history in society forming an entrepreneurial mindset among teachers, students and graduates to thereby achieve national progress with equity, a fact that will contribute to the overall development of universities to society.

Keywords: New university law, social responsibility, public relations. 


\section{Introducción}

Es de conocer que la nueva ley universitaria 30220 trata de reposicionar la misión y la visión de las universidades en el Perú en varios aspectos, principalmente en la investigación y en su responsabilidad social. Bajo este punto de vista, partimos de la premisa de que las universidades son organizaciones que forman profesionales para contribuir con el bienestar y el desarrollo de la sociedad mediante la investigación y la proyección social. Por ende, esto las obliga a involucrarse con la solución de diversos problemas que actualmente aquejan a la sociedad: falta de valores, pobreza, bajo nivel educativo y falta de conocimiento de nuestra realidad nacional.

Si bien es cierto ello, muy pocas veces hubo una reflexión sobre la importancia de la responsabilidad social que deben asumir las universidades y el rol que cumplen sus relaciones Públicas. En este sentido, el objetivo de este artículo es buscar el papel que deben asumir las universidades mediante las relaciones públicas que están contempladas en algunos artículos de la nueva ley universitaria. Por ello, según Pérez (1996, p. 29) “Las relaciones Públicas son fundamentales para mantener un clima de confianza entre todo tipo de organizaciones con la sociedad mediante una comunicación estratégica y acciones que contribuyan al desarrollo humano". Es decir, las Relaciones Públicas se aplican para todo tipo de organizaciones que deseen ser aceptadas en la sociedad. Dicha aceptación garantiza el crecimiento y desarrollo, respaldadas por la denominada licencia social, ninguna organización, por mejor infraestructura que pueda tener si no cuenta con un buen concepto en la sociedad, no podrá subsistir.

\section{Ley Universitaria 30220 y responsabilidad social}

La nueva ley universitaria 30220, muestra la existencia de artículos muy puntuales que hacen notar la importancia del cumplimiento de la responsabilidad social. El artículo $1^{\circ}$ se establece:

El objeto de la ley es el de promover el mejoramiento continuo de la calidad educativa de las instituciones universitarias como entes fundamentales del desarrollo nacional, de la investigación y de la cultura.

Esta afirmación reconoce a las universidades peruanas como entidades que tienen una gran responsabilidad para con el país, ya que por medio de la promoción de la investigación son artífices para contribuir con el mejoramiento 
del nivel intelectual de la sociedad. Toeffler afirma que: "aquella sociedad que tenga conocimiento tendrá el poder". Por ende elevar el nivel educativo y la cultura general garantiza que el desarrollo se haga con más justicia y equidad haciendo posible el acceso al conocimiento de las mayorías, lo cual significa terminar con la elitización de la educación superior. Aquí radica la esencia de las universidades al tener esta noble labor de contribuir con la sociedad del conocimiento.

El artículo $3^{\circ}$ de la ley afirma que:

La universidad es una comunidad académica orientada a la investigación y a la docencia, que brinda una formación humanista, científica y tecnológica con una clara conciencia de nuestro país como realidad multicultural. Adopta el concepto de educación como derecho fundamental y servicio público esencial. Está integrada por docentes, estudiantes y graduados. Participan en ella los representantes de los promotores, de acuerdo a ley.

Llama la atención el énfasis que se pone en el término de "comunidad", lo cual quiere decir que las universidades deben manejarse de manera democrática en donde todos sus integrantes: promotores, directivos, docentes, estudiantes y egresados puedan aportar equitativamente en el desarrollo académico, económico y de programas de responsabilidad social. El hecho de impartir un servicio público educativo la convierte en una expresión de responsabilidad social, porque las universidades asumen una misión muy importante como es el de formar profesionales que no solo se limiten a repetir lo que hayan podido aprender y experimentar en las aulas, sino que por sobre todas las cosas, fomenten la producción de nuevos conocimientos mediante la investigación científica.

La falta de investigación y generación de nuevas tecnologías, hace que el Perú se convierta en un importador de tecnología. Por ello, cuán importante es revertir esta situación tratando de llevar no solo conocimiento, sino creación de conocimiento a todas las regiones del país.

Esta proyección requiere de la labor de relaciones públicas, Kovács (2007, p. 9) menciona que las relaciones públicas consisten en:

una profesión dinámica que tiene por objeto armonizar los intereses de las organizaciones junto con los intereses sociales de los públicos, y ante esta tendencia, garantizar el contacto humano entre nosotros. Este equilibrio de intereses se logra mediante una comunicación franca, sincera, veraz y solidaria que permite a las organizaciones de hoy sensibilizarse mejor con las problemáticas que quejan a nuestra sociedad. 
Según esta definición, las organizaciones, cualquiera que sea su naturaleza, deben buscar dialogar y conocer las necesidades, expectativas, problemáticas y la forma de pensar de diversos públicos, sean internos o externos que se encuentren en la sociedad.

Es más, este punto es ampliado por la nueva ley universitaria en su artículo $5^{\circ}$, numerales $8,9,10,11,15$ y 16 cuando afirma que las universidades deben resaltar los siguientes elementos para su desempeño:

El pluralismo, tolerancia, diálogo intercultural e inclusión. La pertinencia y compromiso con el desarrollo del país. La afirmación de la vida y dignidad humana. El mejoramiento continúo de la calidad académica. La pertinencia de la enseñanza e investigación con la realidad social. El rechazo a toda forma de violencia, intolerancia y discriminación.

Este artículo, hace referencia a que las universidades deben promover el diálogo y desarrollar sus programas educativos de acuerdo con las necesidades y la realidad del entorno social a las que pertenecen para que la población beneficiada se sienta identificada plenamente con estas instituciones. De alguna manera la integración entre universidades y su comunidad contribuye al fortalecimiento del prestigio porque al ser aceptadas por la población se tendrán estudiantes, docentes y personal administrativo más comprometido con la investigación y la búsqueda de soluciones que la sociedad requiera suplir.

El mejoramiento de la calidad académica radica en lograr que el aprendizaje sea significativo para la comunidad universitaria al lograr que todos sus miembros tomen conciencia de la importancia del diálogo desde la concepción de la currícula hasta las estrategias de enseñanza y aprendizaje

Según esta perspectiva, la única manera de que nuestra sociedad pueda progresar es contando con profesionales idóneos que tengan una preparación académica de buen nivel, tanto en conocimientos como en compromiso social, capaces de actuar con ética y valores anteponiendo el bienestar de la colectividad antes que los intereses personales que pueden conducir a los actos de corrupción. Tal como lo manifestó Castro (1998 p. 213)

Cada profesión y cada profesional adquieren mayor dimensión relacionados con valores. Estos pueden enriquecer o hacer despreciable los actos del profesional, según la calidad que tengan. De hecho, no hay acción moral desprovista de relación estrecha y directa con algunos valores; tampoco, profesión sin valores. 
Esto hace alusión a que no existe profesional sin ética. La universidad por ende, es responsable también de inculcar valores a sus estudiantes y docentes. Por esta razón la ley universitaria, en su artículo $6^{\circ}$, numerales 2 y 3 hacen mención a los fines de la universidad:

Formar profesionales de alta calidad de manera integral y con pleno sentido de responsabilidad social de acuerdo a las necesidades del país. Proyectar a la comunidad sus acciones y servicios para promover su cambio y desarrollo.

Formar de manera integral resulta ser un pilar fundamental en la misión que las universidades asumen, en donde claramente se establece que la mejor contribución que ellas puedan hacer a la sociedad es mediante una enseñanza basada en valores y en un alto grado de sensibilidad social, hecho que los obliga a ser responsables, veraces, éticos, solidarios y buscadores de soluciones justas. Ahora ya está incluida en la evaluación de las asignaturas de todas las carreras en el país el rubro de proyección social, hecho que permite a los estudiantes y egresados a tener mayor sensibilidad social y asumir un rol activo en la búsqueda de soluciones viables mediante el conocimiento y la responsabilidad social que las universidades asumen en este aspecto.

Pérez (1996, p. 32) sostuvo la importancia de que todas las organizaciones que deseen lograr la aceptación social deben desarrollar la política del "kimono abierto", es decir, permitir que la sociedad conozca la misión, visión, forma de gestionar y gobernar a las universidades, quiénes integran la junta directiva y la plana docente, la calidad de sus egresados, sus métodos de enseñanza y aprendizaje, la gestión educativa en cuanto a presupuesto y convenios. Una organización que mantiene en secreto sus cifras es una organización sospechosa, ya que al no informar adecuadamente a la sociedad sus logros y dificultades, puede ser víctima de rumores que pueden destruir su reputación. Aquí entran a tallar nuevamente las relaciones públicas, porque al fomentar el diálogo entra las organizaciones y la sociedad busca la sintonización de intereses, y comunicar las acciones que desarrollan a la sociedad.

Las universidades gana prestigio si rinden cuentas claras a la sociedad, explican sus programas educativos y sus métodos, pero por sobre todo, si desarrollan actividades de responsabilidad social presentando sus resultados mediante un balance social. Ninguna entidad en la actualidad puede buscar ser confiable si no comunica con claridad sus acciones y logros, así como también si no expone con precisión sus políticas y sus procesos de hacer las cosas. Ello requiere transparencia en la gestión educativa que desarrollan las universidades. 
La ley nuevamente hace notar esto en su artículo $11^{\circ}$, numerales del 1 al 6 al afirmar que:

\begin{abstract}
Las universidades públicas y privadas tienen la obligación de publicar en sus portales electrónicos, en forma permanente y actualizada, como mínimo, la información correspondiente a: - El Estatuto, el Texto Único de Procedimientos Administrativos (TUPA), el Plan Estratégico Institucional y el reglamento de la universidad. - Las actas aprobadas en las sesiones de Consejo de Facultad, de Consejo Universitario y de Asamblea Universitaria. - Los estados financieros de la universidad, el presupuesto institucional modificado en el caso de las universidades públicas, la actualización de la ejecución presupuestal y balances. - Relación y número de becas y créditos educativos disponibles y otorgados en el año en curso. - Inversiones, reinversiones, donaciones, obras de infraestructura, recursos de diversa fuente, entre otros. - Proyectos de investigación y los gastos que genere.
\end{abstract}

Las universidades están obligadas a presentar con total transparencia todo lo concerniente a su gobierno, quiénes la dirigen, cuántos ingresos tienen, en qué invierten dichos ingresos, las decisiones que se tomen en consejos universitarios y de facultad respecto a beneficios como las becas y los créditos educativos. A medida que las universidades muestren a la sociedad la gestión que éstas desarrollan y expliquen de manera transparente las razones de sus acciones y políticas, generarán confianza y comprensión por parte de la comunidad universitaria misma y de la sociedad en general. La nueva ley inclusive va más allá de la simple presentación de los balances económicos de las universidades al obligarlas a mostrar la partida presupuestal para los proyectos de investigación que resulten beneficiosos para el desarrollo de las comunidades que rodean a las universidades. Evidentemente se trata de efectuar relaciones públicas concretas mediante aportes y conocimientos que permitan prestigiar a las universidades por un lado y contribuir con el desarrollo de la sociedad por el otro, lo cual hace posible que la misión y visión de las universidades estén en perfecta armonía de intereses con la sociedad.

Es decir, las universidades a la luz de esta ley se convierten en protagonistas del desarrollo social porque aportan con propuestas viables de solución a uno de los principales problemas de nuestra sociedad: la educación y la formación de profesionales que tengan valores y que estén comprometidos con el crecimiento justo de nuestra comunidad. Para ello es que la nueva ley universitaria considera como factor primordial para este logro a los institutos de investigación cuando en su artículo $30^{\circ}$ manifiesta que: 
La existencia de Institutos de Investigación en las universidades se considera un criterio favorable para el proceso de acreditación de su calidad.

Las universidades a la luz de esta nueva ley, deben tomar a la investigación científica como la razón de ser de las mismas, ya que ahora no basta solo con repetir el conocimiento adquirido por otros investigadores fuera de nuestra realidad, hecho que quizás sea el factor causal del por qué nuestros egresados no responden adecuadamente a las necesidades de nuestra sociedad al seguir modelos de enseñanza y aprendizaje que no están acordes con nuestra realidad, sino que es necesario que dichos conocimientos sean hechos por nuestros docentes, estudiantes y egresados de acuerdo con nuestra realidad nacional, llegando a las comunidades más alejadas de nuestro país y permitiendo a la vez que dichas comunidades también tengan acceso a dicho conocimiento y que lo puedan aplicar respetando sus culturas, costumbres y cosmovisiones, es decir, respetando su dignidad humana. Esto es citado en el artículo $48^{\circ}$ al afirmar que:

La investigación constituye una función esencial y obligatoria de la universidad, que la fomenta y realiza, respondiendo a través de la producción de conocimiento y desarrollo de tecnologías a las necesidades de la sociedad, con especial énfasis en la realidad nacional.

Las relaciones públicas por su parte, según esta nueva ley, son aludidas de manera indirecta porque entre sus funciones está que las organizaciones, entre ellas las educativas, deben fomentar la apertura, el diálogo y la búsqueda de oportunidades que les permita a sus públicos, internos y externos, obtener beneficios para su bienestar y desarrollo. Kovács $(2002$, p. 35) menciona: "Las organizaciones de hoy no deben ver a su competencia como enemiga, sino más bien como una posible aliada estratégica que les permita optimizar sus servicios y brindar así una mejor calidad de vida a la sociedad". Una sociedad se hace grande y próspera por la calidad de su gente, y en ello las universidades tienen mucho por hacer. El artículo $51^{\circ}$ es claro en esto porque de manera imperativa señala que:

Las universidades coordinan permanentemente con los sectores público y privado, para la atención de la investigación que contribuya a resolver los problemas del país. Establecen alianzas estratégicas para una mejor investigación básica y aplicada. 
Esto a su vez es complementado con el artículo $59^{\circ}$, numeral 13 de la nueva ley universitaria cuando afirma que las universidades deben:

Celebrar convenios con universidades extranjeras, organismos gubernamentales, internacionales u otros sobre investigación científica y tecnológica, así como otros asuntos relacionados con las actividades de la universidad.

Es más, el Estado, según esta nueva ley en su artículo $116^{\circ}$ numeral 4 , promueve la celebración de convenios estratégicos entre las mismas universidades y empresas hasta con la reducción de los impuestos que estas deben pagar al fisco, ya que considera que cualquier aporte en la ciencia y tecnología que favorezca el desarrollo del país se debe reconocer como un hecho plausible merecedora de exoneraciones tributarias como parte de la responsabilidad social que asumen las casas superiores de estudios en bien de nuestra sociedad. Con esto el Estado impulsa la formación de estas alianzas estratégicas para promocionar becas, pasantías y aportes a las publicaciones que contribuyan eficientemente al desarrollo humanístico de los integrantes de la comunidad universitaria, lo cual favorece en prestigio y en economía a las mismas universidades, al establecer que:

Los convenios de cooperación celebrados entre instituciones universitarias y otras personas jurídicas de cualquier naturaleza que tengan por finalidad contribuir a la mejora de la calidad educativa, científica, tecnológica y al desarrollo deportivo del país, gozan de beneficios tributarios, conforme a la legislación pertinente sobre la materia.

Entre los otros asuntos relacionados con la universidad a la cual hace mención esta ley, se incluyen los seminarios, diplomados, intercambios docentes y estudiantiles y certificaciones mancomunadas que prestigien y optimicen la formación de los integrantes de la comunidad universitaria. Estas experiencias resultan ser muy enriquecedoras en la formación de la calidad humana y profesional de los docentes, estudiantes y egresados, con lo cual las universidades pueden lograr ese concepto favorable ante la sociedad que es el fin que propugnan las relaciones públicas aplicadas al sector educativo.

Además de ello, las universidades de hoy no se deben limitar a formar egresados que busquen trabajo en diversas empresas e instituciones, sino que sean capaces de producir su propio puesto laboral y dar oportunidades de trabajo a los demás. De alguna manera el crear empresas es una manifestación de amor por el prójimo porque ello permite que muchos profesionales puedan ejercer y mejorar sus talentos en favor de la sociedad. Para ello las universidades deben ser 
promotoras de iniciativas empresariales, hecho que también es una manifestación de responsabilidad social en bien de la comunidad. Para ello, el artículo $52^{\circ}$ hace alusión a las incubadoras de empresas al mencionar que:

La universidad, como parte de su actividad formativa, promueve la iniciativa de los estudiantes para la creación de pequeñas y microempresas de propiedad de los estudiantes, brindando asesoría o facilidades en el uso de los equipos e instalaciones de la institución.

Uno de los puntos neurálgicos de esta nueva ley universitaria es lo concerniente a la responsabilidad social. Respecto a este punto, es necesario aclarar que en nuestro medio muchas universidades y empresas no han comprendido adecuadamente en qué consiste esta responsabilidad. Partimos de la definición de que responsabilidad tiene que ver con hacerse cargo de algo y asumir como propia las consecuencias de una acción o política. Esto incluye también a las universidades, además de las públicas, principalmente a las privadas que poseen por lo general más recursos que las del Estado.

Esta contribución a la sociedad es lo que genera un concepto favorable de la sociedad hacia las organizaciones empresariales, que en los últimos años han venido valorando con mayor énfasis esta aceptación social como uno de los principales pilares para su respectivo desarrollo. Por ello es que los promotores y miembros de la comunidad universitaria en conjunto deben tomar en cuenta que la responsabilidad social resulta ser un compromiso en donde las organizaciones deben ser conscientes del impacto que pueden generar sus decisiones y acciones en la comunidad.

En el capítulo XIII, en su artículo $124^{\circ}$, se puntualiza con precisión que:

La responsabilidad social universitaria es la gestión ética y eficaz del impacto generado por la universidad en la sociedad debido al ejercicio de sus funciones: académica, de investigación y de servicios de extensión y participación en el desarrollo nacional en sus diferentes niveles y dimensiones; incluye la gestión del impacto producido por las relaciones entre los miembros de la comunidad universitaria, sobre el ambiente, y sobre otras organizaciones públicas y privadas que se constituyen en partes interesadas. La responsabilidad social universitaria es fundamento de la vida universitaria, contribuye al desarrollo sostenible y al bienestar de la sociedad. Compromete a toda la comunidad universitaria. 
Sin embargo, es necesario hacer ver que en nuestro país el término responsabilidad social no es entendido en su real dimensión por todas las organizaciones, por lo que suele ser confundido con una simple Inversión Social. ¿Cuál es la diferencia entre ambos conceptos?

\section{Inversión social vs responsabilidad social}

Existe una confusión entre lo que es inversión social y responsabilidad social. Esto es muy común en nuestros países ya que se piensa equivocadamente que la responsabilidad social solamente consiste en efectuar donaciones, auspicios y mecenazgos que de alguna manera reviertan en mayores ganancias a las mismas empresas. En la inversión social predomina la idea de que si la sociedad percibe que la empresa le otorga ciertos beneficios, entonces la misma comunidad se convertirá en una aliada estratégica que le permitirá a las empresas e enfrentar con éxito situaciones críticas o de riesgo. Es decir que, de manera consciente o inconsciente se piensa en los futuros réditos que puedan generar dichos programas. Ejemplo de inversión social podemos citar al sponsoring o patrocinio a favor de certámenes culturales, equipos deportivos, programas educativos o ayudas temporales en donde aparece abiertamente la marca y el nombre de las empresas que producen ciertos productos y servicios.

\section{Responsabilidad social}

Para que una inversión social se convierta en un verdadero programa de responsabilidad social, lo que falta es asumir un compromiso de liderazgo que pueda generar cambios sociales y que ayuden eficientemente al Estado al logro del bien común. Por ello es que las organizaciones deben tener desde la declaración de su misión, una visión de futuro en conjunto con el bienestar de la sociedad. Es que el bienestar, además de promover acciones como el cuidado sostenido del medio ambiente, la asistencia educativa y de salud para las poblaciones más necesitadas, como lo afirma el relacionista $\operatorname{Norero}(2000$, p. 39) al sostener que "la Responsabilidad Social también incluye promover liderazgos entre los miembros de la comunidad que puedan generar cambios en la mente de la población" , lo cual permitirá que por ellos mismos dejen el atraso y los paradigmas equivocados de dependencia de la caridad, sea del Estado o de las mismas empresas privadas. "Enseñar a la población a pescar y no a pedir pescado" resume metafóricamente el real propósito de la Responsabilidad Social.

Es decir que la nueva ley exige a las universidades a buscar diversos medios para cumplir con su Responsabilidad Social recalcando que ésta es 
un punto a favor para la acreditación institucional cuando demuestren que contribuyen eficientemente en el desarrollo social. Este término de "desarrollo" obliga a que las universidades no se limiten a dar dádivas y obsequios para menguar las necesidades de diversas poblaciones en condiciones de pobreza, ya que después de ello dichos problemas al no solucionarse, no cambian la realidad de estas poblaciones. En cambio con programas reales de responsabilidad social, las universidades mediante sus proyecciones sociales se ven obligadas a asistir y educar a dichas poblaciones para estas sea capaces de autodesarrollarse. Por ello es que el artículo $125^{\circ}$ de la nueva ley universitaria afirma que:

Cada universidad promueve la implementación de la responsabilidad social y reconoce los esfuerzos de las instancias y los miembros de la comunidad universitaria para este propósito; teniendo un mínimo de inversión de $2 \%$ de su presupuesto en esta materia y establecen los mecanismos que incentiven su desarrollo mediante proyectos de responsabilidad social, la creación de fondos concursables para estos efectos. El proceso de acreditación universitaria hace suyo el enfoque de responsabilidad social y lo concretiza en los estándares de acreditación, en las dimensiones académicas, de investigación, de participación el desarrollo social y servicios de extensión, ambiental e institucional, respectivamente.

\section{Conclusión}

Las universidades peruanas ya no pueden limitarse a ser caritativas obsequiando alimentos, computadoras u otros implementos para el bien de las comunidades sin enseñarles a dichas poblaciones cómo manejar los nuevos conocimientos y tecnologías, sino que deben aspirar a convertirse en lideresas proactivas que generen cambios que promuevan una sociedad libre de violencia, drogadicción, alcoholismo, corrupción y la delincuencia común, el trabajo en conjunto entre las universidades con los miembros de la comunidad social y universitaria además de los gestores de la comunicación, principalmente los relacionistas públicos en su calidad de nexos entre las organizaciones educativas y la sociedad. La nueva ley universitaria, se coloca como un marco que permite el nexo entre la investigación y la comunidad, esperemos este desarrollo. 


\section{Referencias}

Gobierno del Perú (2014). Nueva Ley Universitaria 30220 (2014) Diario Oficial El Peruano. Normas legales 12914. Lima: Perú. Edición del miércoles 9 de julio de 2014.

Castro, R. (1998). Ética profesional. Lima: Perú. Editorial Universidad de San Martín de Porres. P. 213

Kovács, I. (2007). Relaciones Públicas: Ética, valores y teorías. Lima: Perú. Editorial Universidad Peruana Unión.

Kovács, I. (2002). Relaciones Públicas: Del presente al futuro. Lima: Perú. Editorial Alberto Díaz.

Pérez S, (1996). XXI Congreso Internacional de Relaciones Públicas. Santiago de Chile.

Norero L. (2000). Responsabilidad Social y Relaciones Públicas. Tesis de grado no publicada. Universidad de San Martín de Porres. 